EMBRYARIDDLE
Aeronautical University

SCHOLARLY COMMONS

\section{International Journal of Aviation,} Aeronautics, and Aerospace

\title{
Enhancing Quality Assurance using Virtual Design Engineering: Case Study of Space Shuttle Challenger
}

Kouroush Jenab

Embry-Riddle Aeronautical University, k.jenab@moreheadstate.edu

Scot Paterson

Embry-Riddle Aeronautical University, thesazi@gmail.com

Follow this and additional works at: https://commons.erau.edu/ijaaa

Part of the Aerospace Engineering Commons, Atmospheric Sciences Commons, and the Meteorology Commons

\section{Scholarly Commons Citation}

Jenab, K., \& Paterson, S. (2015). Enhancing Quality Assurance using Virtual Design Engineering: Case Study of Space Shuttle Challenger. International Journal of Aviation, Aeronautics, and Aerospace, 2(4). https://doi.org/10.15394/ijaaa.2015.1071

This Article is brought to you for free and open access by the Journals at Scholarly Commons. It has been accepted for inclusion in International Journal of Aviation, Aeronautics, and Aerospace by an authorized administrator of Scholarly Commons. For more information, please contact commons@erau.edu. 


\section{Enhancing Quality Assurance using Virtual Design Engineering: Case Study of Space Shuttle Challenger}

\section{Cover Page Footnote}

The authors would like to express their sincere appreciation to anonymous referees for their valuable comments which enhanced the quality of this paper. 
Many Quality Assurance (QA) techniques require the QA team to predict a particular hazard before it can be categorized and managed (Rausand, 2005). On top of this, standard QA techniques generally fail to consider multiple or complex hazard interactions. These interactions lead to situations where multiple minor hazards could interact to create a catastrophic outcome (Rausand, 2005). Virtual Design Engineering allows for the exploration of a design and its potential failure modes before it has even been built. Expanding upon this capability, QA engineers can utilize the massive computing power available today to stimulate and simulate various failure modes and their interactions. The virtual environment would allow the engineer to study extremely difficult situations with respect to quality and hazards by virtually testing various solutions in a cost effective manner. For example, a QA engineer could use the known physical characteristics of the materials in the Space Shuttle Solid Rocket Booster (SRB), apply extreme environmental factors such as low temperature, and extreme wind shear forces, and simulate the pressurization of the system. By utilizing Virtual Design Engineering processes, the engineer could pause time and virtually explore the entirety of the system, discovering any potential hazards and quality issues.

The SRB as used on the Challenger were comprised of 11 individual sections approximately 12 feet in diameter that were fitted together using tang-andclevis joints secured by 177 steel pins as demonstrated in Figure 1 from the Rogers Commission Report (Rogers Commission, 1986). Sections of the SRB are joined together at the factory to reduce the number of joints to be fitted by engineers at the assembly building to four, known as "field joints" (Rogers Commission, 1986).

The Rubber O-Rings are coated with a Zinc Chromate Putty to act as a kind of insulation between the hot gasses and the O-Rings themselves, however this putty was also integral in the "activation and seal" of the O-Rings (Rogers Commission, 1986). When the putty was pressed outward it would act to force the O-Ring into the gap between the Tang and Clevis joint in a process called "pressure activation of the O-Ring seal" (Rogers Commission, 1986). Should this pressure activation be delayed, for example by cold temperatures, the gap could be opened considerably and there would be a high probability that the hot gasses would escape past the O-Ring and "damage or destroy" the seals (Rogers Commission, 1986). Many tests of the O-Rings have shown resiliency degradation due to "low to moderate temperatures", and thus they were unable to achieve the proper activation and seal in the required time of 600ms (Rogers Commission, 1986). 


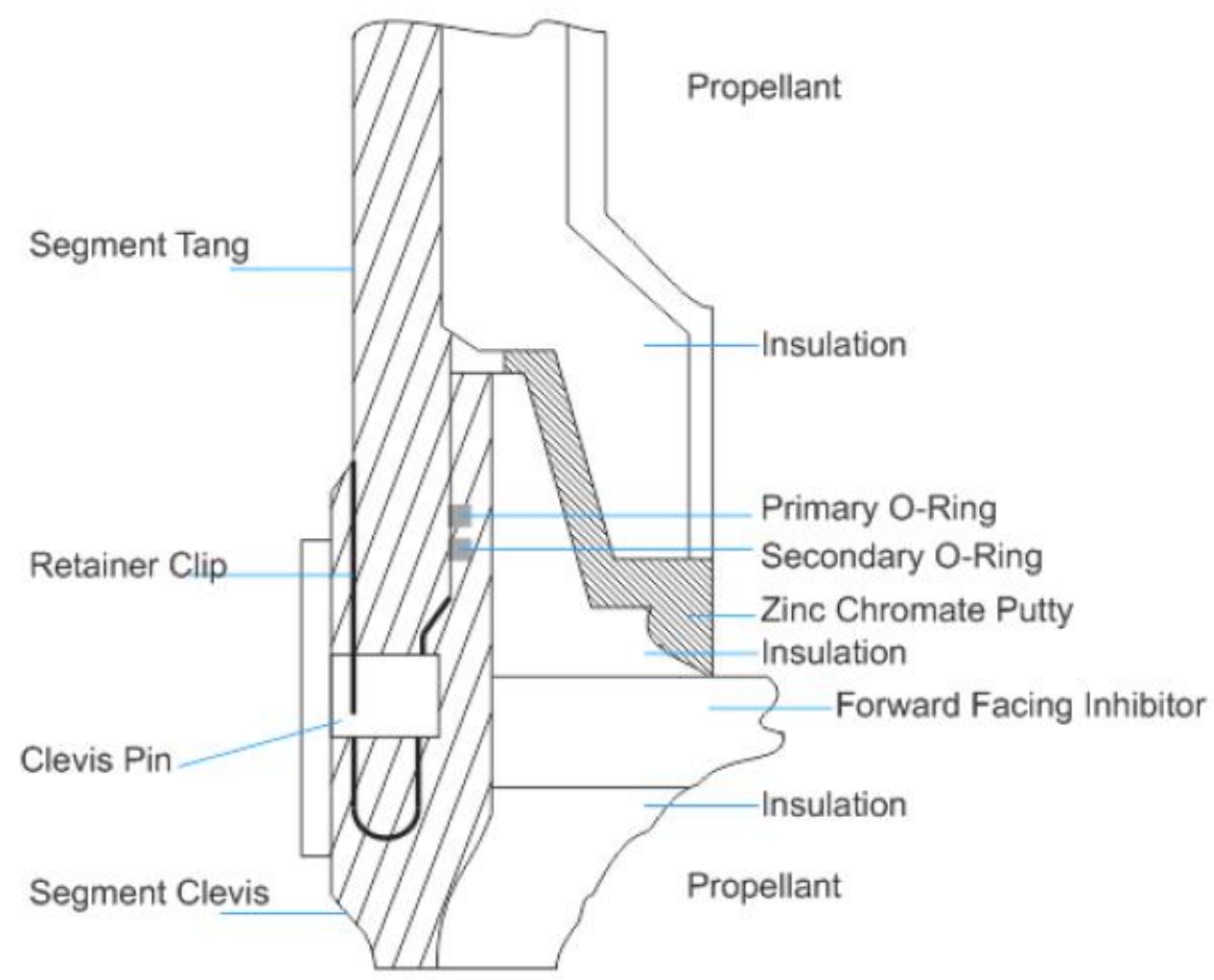

Figure 1. Tang-and-clevis connection (from Jenab \& Pineau, 2015)

Eventual failure of the joint within the SRB was due to a combination of these complex factors, but of particular importance were the inadequate O-Ring seal and the interaction of the eventual escaping hot gasses with the aerodynamic forces of the spacecraft as it ascended through the atmosphere (NASA, 1986). As the space shuttle ascended it encountered wind shear effects matching the largest values experienced on previous flights, which created a relatively large fluctuation of forces on the vehicle and potentially magnified any existing defects (NASA, 1986). At 58.788 seconds into the flight, the first flame was captured on video, which grew into a "continuous, well-defined plume" shortly thereafter (NASA, 1986). As this flame grew "it was deflected rearward by the aerodynamic slipstream and circumferentially by the protruding structure of the upper ring attaching the booster to the External Tank" (NASA, 1986). Within only a matter of seconds this flame, directed by the complex aerodynamic forces of ascent, impacted and breached the external fuel tank leading to the catastrophic loss of the spacecraft and crew (NASA, 1986). Using a Virtual Reality QA model would help the QA engineers detect the interactions of known hazards during the extreme 
environmental conditions experienced on launch morning, giving them a high risk level and forcing their mitigation prior to any actual failure with the launch vehicle.

\section{Literature Review}

Virtual Reality (VR), also known as Virtual Environment (VE), refers to an "artificial reality" created utilizing computers to give the user a "first-person, interactive view into the simulated (hypothetical) world that has been created" (Lerner \& Lerner, 2013). Virtual Manufacturing (VM) takes this one step farther within the manufacturing sector, in that a computer system is utilized to generate information related to the "structure, status, and behavior" of a particular system within a virtual environment (Mujber et al., 2004). The end goal with VM is to manufacture the system within the computer simulation environment and discover manufacturing and assembly difficulties prior to actually physically building the system.

The natural extension of this is Virtual Prototyping (VP), which is taking the virtually manufactured system and applying a real world environment to it within the computer simulation to see how it reacts under real-world operating conditions (TWI, 2014). VP allows an engineering team to design, build, and apply realistic tests to the system both mathematically and visually (TWI, 2014). Many industries utilize VP in this way to include automotive, oil/gas, shipbuilding, aerospace, and defense (TWI, 2014). For example, automobile manufacturers can create their vehicle within a virtual environment and virtually crash it to ensure it will meet government crash test standards far before any part of the vehicle is actually produced. One such company to recently take full advantage of these abilities was Fisker Automotive. The Fisker Karma was a plug-in hybrid that utilized a special sub frame to house both a fuel tank and electric motors (Chou \& Üllrich, 2013). The Fisker Karma engineering team built the entire rear of the car in a virtual environment and verified it would pass crash tests before being built (Chou \& Üllrich, 2013). Putting the system through real world scenarios in this way allows one to visually portray the results in a generally easy to understand way, with most software packages providing at least some level of photorealistic rendering capability (Hudspeth, 2005).

VR systems can generally be classified into one of three main categories; non-immersive (desktop, laptop), semi-immersive (projector systems), and fully immersive (VR goggles/glasses) (Mujber et al., 2004). The most basic form of VR representation is the non-immersive method. This places a user within the 3-D environment while sitting at a conventional workstation with a monitor, keyboard, and mouse (Robertson et al., 1993). All of the 3-D cues and interaction found in the 
other VR representations are found here, however the viewer is limited in scope to just the screen on the workstation (Robertson et al., 1993).

A significantly more involved VR representation method is the semiimmersive method. A semi-immersive method gaining traction has been the Cave Automatic Virtual Environment (CAVE), which is essentially a theater in a room (Nesbitt, 2013). This setup requires multiple projectors displaying on each individual wall or it could consist of multiple projectors displaying on a rounded dome (Nesbitt, 2013). Controls include utilizing a joystick or manual input device or utilizing motion tracking technology to track the user and move around within the virtual environment based upon the movements of the user (Nesbitt, 2013). The addition of special 3-D glasses allows the user to have the feeling of full immersion within the virtual environment (Nesbitt, 2013). Advancement in CAVE technologies have allowed Brown University to create an environment with one arc-minute resolution and head tracked stereo sound with a nearly seamless screen (Kenyon et al., 2014). A graphic representation of Brown University's CAVE is shown in Figure 2.

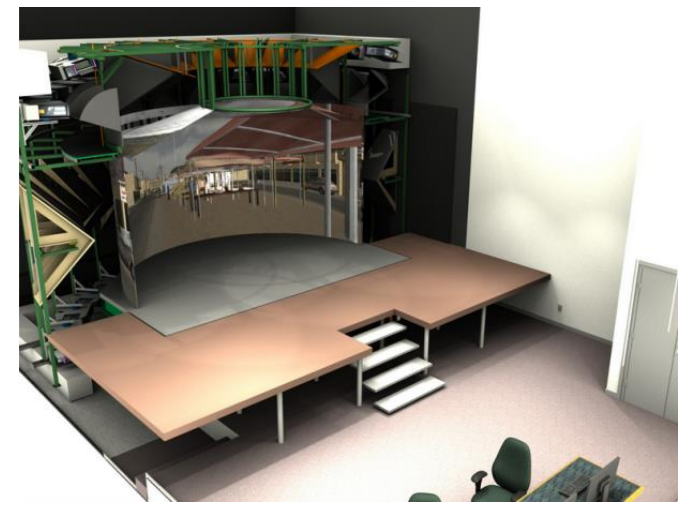

Figure 2. Brown University's CAVE (from Kenyon et al., 2014)

Fully immersive VR typically consists of a head mounted display (HMD) made up of two separate display screens mounted to a helmet or glasses, which the user wears (Takashi, 2002). Fully immersive VR via the HMD has the benefit of having a significantly smaller footprint in comparison to the CAVE methods discussed above, however it has proven difficult to achieve miniaturization, high resolution, and a wide field of view all at the same time (Takashi, 2002). The closeness of the screens to the user's eyes creates a mismatch between the accommodation and the convergence, which might contribute to visual fatigue often associated with this type of display (Takashi, 2002). In addition, adding this extra weight can increase the stress the user's neck bears, resulting in additional 
fatigue (Knight \& Baber, 2007). Smaller and lighter HMD units are becoming increasingly commercially available, which has made them cheaper and easier to use. One such device is the Oculus Rift, which totes a 100-degree field of view combined with realistic parallax, scale, and depth all in a lightweight and cost effective package (Oculus VR, n.d.).

Various QA techniques have been utilized over the years as a part of the Failure Mode, Effectives and Criticality Analysis (FMECA) process, particularly the Preliminary Hazard Analysis (PHA), System Hazard Analysis (SHA), and Fault-tree Analysis (FTA). PHA typically does not go into extensive detail, as by its very nature it is preliminary. This type of analysis is utilized to discover system level hazards and risks early in the development to help influence design decisions to avoid costly mistakes. It is during this PHA a preliminary understanding of potential hazards, causes, and risks associated with the system are documented. Identification of "Safety Critical Functions (SCF)" is a key element to the PHA (Ericson, 2005). A major difficulty with the PHA is that it is difficult to recognize the effects of interactions between hazards and hazards generally must be foreseen by the analysis (Rausand, 2005).

SHA uses the PHA as a baseline and expands its findings (Safeware Engineering Corporation, 2003). By starting with the previously developed PHA, the SHA "considers the system as a whole" and looks to find how the system's operation, subsystem interfaces and interactions, operator interactions, and component failures contribute to overall system hazards (Safeware Engineering Corporation, 2003). The overall goal is to "identify and assess system-level hazards", which typically consist of hazards associated with "the interfaces and interactions between subsystems" (Redmond, 2007). The primary output of this SHA is a list of hazards along with the assessment of the risk associated with the hazard (Redmond, 2007). An example table utilized to classify these hazards based on risk is included in Figure 3 (Koopman, 2011). SHA could be greatly enhanced by the use of VR and VP as described above.

\begin{tabular}{|c|c|c|c|c|c|c|}
\hline \multicolumn{2}{|c|}{ Risk Table } & \multicolumn{5}{|c|}{ Probability } \\
\cline { 3 - 7 } & & Very High & High & Medium & Low & Very Low \\
\hline \multirow{2}{*}{$\stackrel{\text { Very High }}{ }$} & Very High & Very High & Very High & High & High \\
\cline { 2 - 7 } & High & Very High & High & High & Medium & Medium \\
\cline { 2 - 7 } & Medium & High & High & Medium & Medium & Low \\
\cline { 2 - 7 } & Low & High & Medium & Medium & Low & Very Low \\
\cline { 2 - 6 } \\
\cline { 2 - 6 } & Very Low & Medium & Low & Low & Very Low & Very Low \\
\cline { 2 - 7 }
\end{tabular}

Figure 3. Example Risk Table 
An FTA can run either forward (inductive) or in reverse (deductive) starting from an "undesired event" and working backwards to find the basic causes or working from basic causes to find their eventual outcome (Vesely, 2005). Generally speaking, a quality issue/hazard/risk is defined and resolved into its contributing factors; this applies again to each factor until the basic causes are identified (Vesely, 2005). When laid out, these diagrams form what is referred to as the "fault tree" (Vesely, 2005).

It has been proposed that VP be utilized in system QA verification tests (McGarry et al., 2005). For example, one might develop a virtual prototype of an electrical system and apply various factors to the system to verify the expected results, or to ensure previous QA measures functioned properly. This verification of QA techniques both saves money and time, as one does not need to wait until an end product of some type is built to test and then implement changes. These changes can be put into the virtual model and retested quickly.

VR and VP would greatly enhance the already established QA techniques by making it easier to find hazards/quality/risk factor, discover and assess the interaction of multiple hazards, apply hazard controls, and document potential hazards in an easy to understand way so that everyone from management to the engineering team fully understands the stakes involved.

\section{Virtual Design Engineering Methodology to Enhance Quality Assurance}

Typically Virtual Design Engineering is used to verify a design and the QA process. Virtual Design Engineering should be utilized for more than just the verification, but as the primary analysis method through the QA process. This model would be called the Virtual Reality Quality Assurance (VRQA) model (Figure 4).

The VRQA model starts with a defined scenario, such as a system within an environment. This environment is mapped using VP tools to include the individual components constructed from various commercial off the shelf (COTS) tools, such as those from Autodesk. Environmental variables would then be applied to those components prior to their integration into the virtual environment.

The virtual environment is made up of the damage propagation model and a damage estimate model database. Material physics are applied to the components with respect to their previously applied environmental factors and an experimental fault tree is stepped through using these results. A damage estimate model database is then used to apply potential damage probability and severity to the model and the 
scenario results are generated. Scenario results would then be compared by the software to a baseline set of results and differences would be bookmarked for viewing by a QA engineer.

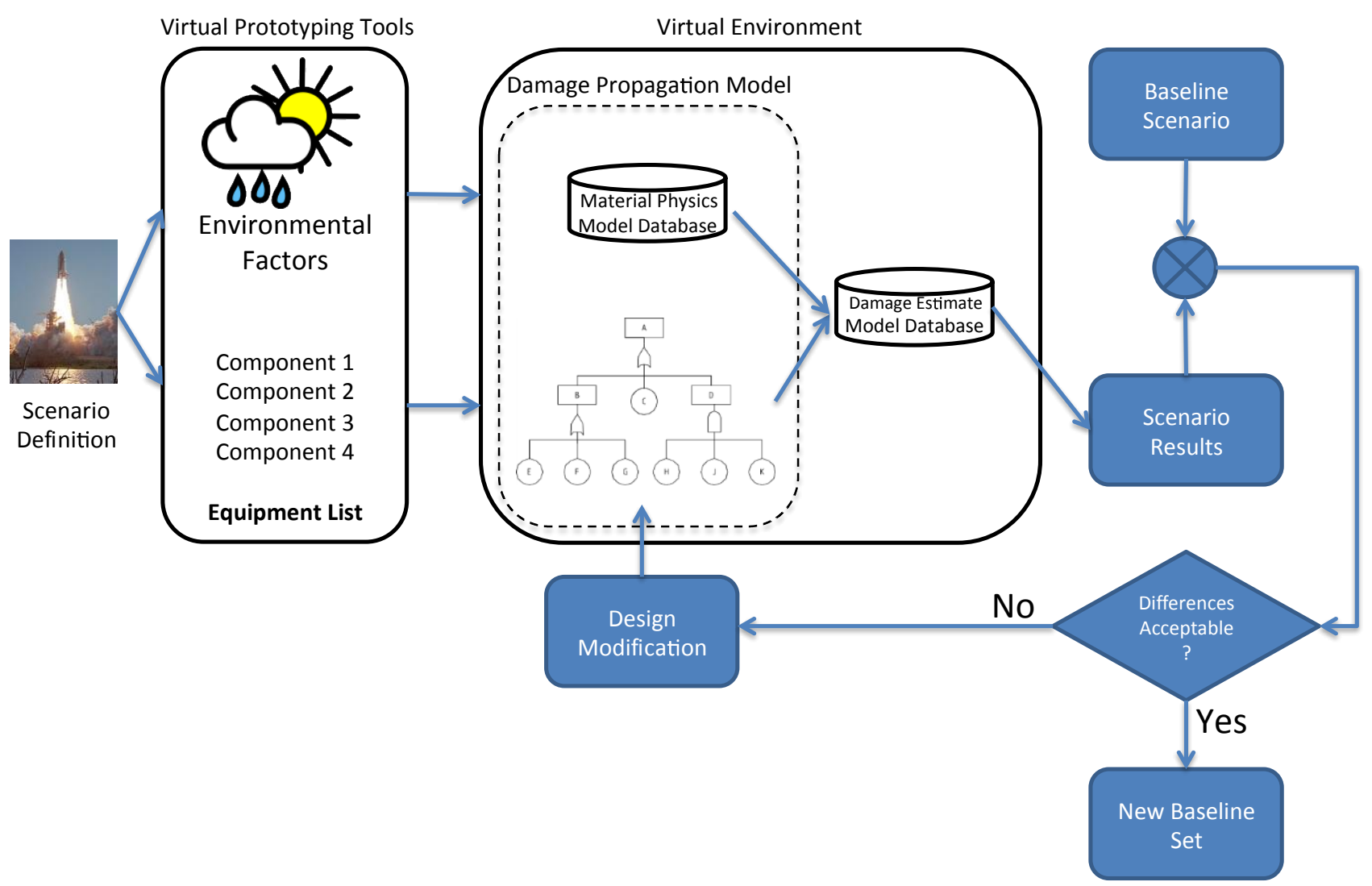

Figure 4. Virtual Reality Quality Assurance Model

These results are within the 3D virtual reality workspace and can be stepped through time for a predetermined interval within a defined resolution. The operator can switch between the two virtual worlds to graphically see the difference between the two and decide if the modifications to the system are satisfactory, and if not they can be targeted for further system modification and testing. Surfaces of objects should have the ability to be color coded for various physical factors, such as increased heat, stress, or decreased structural integrity for the operator to visually reference.

If the simulated results are deemed satisfactory, the output scenario results would be saved as the new baseline for all future modifications to be compared to. An illustrative example of the VRQA model analysis via FTA is found in Figure 5. 


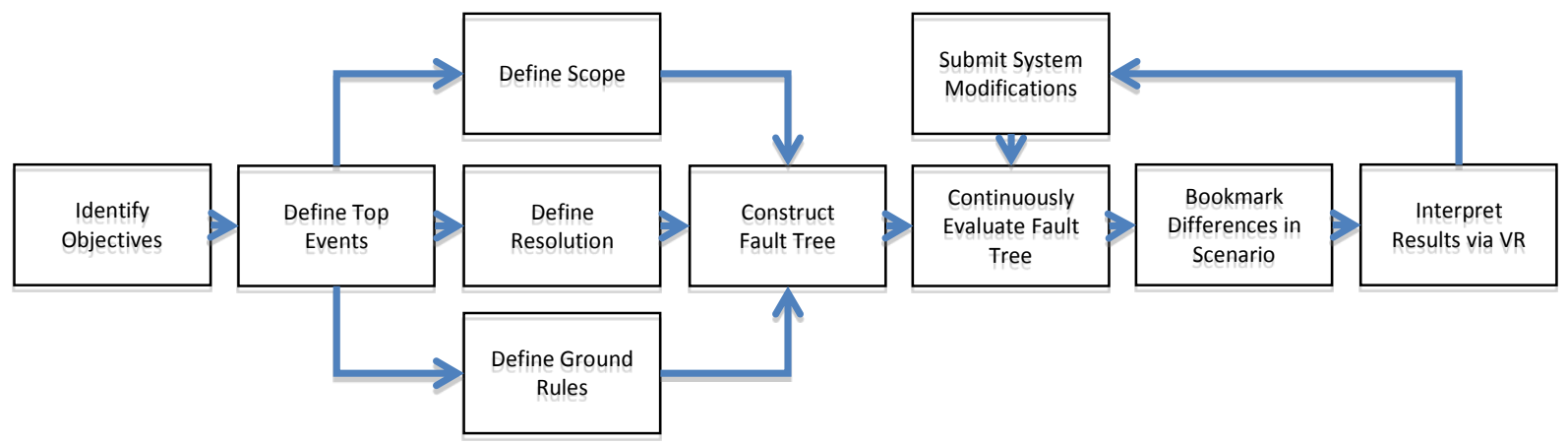

Figure 5. Fault Tree Analysis with Virtual Reality Quality Assurance Methodology

The beginning of the fault tree analysis is virtually unchanged from the traditional methods. First one must identify the objectives of the analysis or the focus of the fault tree. From there the top events or system failure must be determined. The system's boundaries must be determined to define the scope of the simulation, along with the resolution and ground rules. The higher the resolution, the more detailed the simulation must be. For example, are we to treat individual components as solid objects for the sake of simulation or are we going to construct the insides of the components along with each piece's physical make up? The expected initial fault tree can then be constructed using traditional methods.

The fault tree can then be evaluated within the virtual world using VR. This whole process should occur as early in the project's development as possible so a baseline configuration can be generated. This baseline configuration can then be compared with new scenarios generated after development modifications have been applied or later after system completion when new conditions have been discovered which the original fault tree hadn't been tested for. During these comparisons, differences between the new simulation and the baseline simulation can be bookmarked within the virtual environment for a QA engineer to interpret. Modifications based upon these interpretations can be introduced into the system and a new simulation on the fault tree can be evaluated and compared against the baseline. Once these newly introduced system modifications have met quality standards, they will become part of the new baseline for which all-subsequent modifications or environmental affects will be compared to.

\section{Illustrative Example}

The utilization of the Virtual Design Hazard Analysis model during the development of the Space Shuttle system may have potentially avoided the 
catastrophic events that occurred on that cold January morning. Virtual Design may have assisted the QA teams in discovering during the early design stages that the SRB Tang and Clevis joint was flawed and needed a significant redesign. The Rogers Commission discovered that the Thiokol design was based heavily upon reusing design ideas from the Air Force's Titan III solid rocket. Although it was indeed based heavily on that Titan III design, it differed in what would later become extremely key areas. Thiokol engineers would later attempt to compensate for these changes with the addition of the secondary O-Ring, however they did not anticipate their changes causing the overall shell of the rocket motor to warp and deform in an unexpected manor, rendering the O-Rings and their associated Zinc Chromate Putty less effective (NASA, 1986). They also didn't anticipate the changes in the O-Ring specification - from one solid ring in the Titan III to five sections glued together in the SRB - to have as much of an impact as it did (NASA, 1986). Utilizing the Virtual Design Hazard Analysis model the engineers could have tested their design changes and compared them to the baseline simulation of the rocket motor in the Titan III before any pieces of the SRB had actually been built.

To give the project a baseline simulation to compare to, the development team could have built a Titan III model along with its original fault tree analysis within the virtual environment. This original model could have been key, since the team decided to modify the Titan III rather than break new ground on a total redesign. The team could then design their modifications towards the eventual SRB design and submit them for their inclusion within the virtual environment. The new modified simulation results including data from the damage propagation model and the damage estimate model could then be compared to the baseline model and differences may have been bookmarked by the computer for later interpretation and inspection by a member of the QA team.

This graphical representation of results from a modification to the original design may have shown flames shooting upward and then redirected downward towards the external fuel tank by aerodynamic pressures, a complex hazard with multiple interactions best understood by actually witnessing the event unfold. This discovery of significantly more complex hazard interactions may have identified an unknown or misunderstood risk or even moved the multiple previous known risks into a higher category. A static leak of hot gasses upwards from the joint might have had some risk, but the introduction of a redirecting force bringing those hot gasses down towards the external fuel tank may have clearly increased the overall associated risk. Virtual Design Hazard Analysis might have made this interaction exceedingly clear. 
The VRQA model also has the potential to alleviate potential human factors as a system risk. According to the Challenger report, many engineers had knowledge of various O-Ring seal issues however most of their concerns were disregarded or ignored prior to the incident. Significant problems came from the management of both Thiokol and NASA, who "accepted escalating risk apparently because they "got away with it last time"" (NASA, 1986). This tendency to accept unnecessary risk could be tempered by visually displaying the hazard of record along with its simulated expected outcome. The VRQA model allows one to manipulate environmental factors prior to running a simulation, allowing the engineers at the time to quickly simulate the temperatures never experienced before and comparing their results to the baseline results. This process could then be used to create photorealistic exhibits of the hazard in action and make ignoring an issue simply because they got "lucky" significantly more difficult to defend.

\section{Conclusion}

The field of QA is an ever changing discipline chasing an ever more difficult ideal - to mitigate and manage all potential hazards to a given system. As systems become even more complex, this ideal becomes all the more difficult to attain. Virtual Design Engineering is an ever expanding field which could potentially revolutionize QA on large complex projects by bringing to light potential issues before they fully develop in an easy and straight forward manor, saving both resources and human capitol. Photorealistic artifacts from VRQA model simulations can be used to help improve the eventual design as well as get nonengineer types on board with difficult to understand issues, making sure management stays within the loop and fully informed and ensuring unnecessary risks are mitigated or avoided. At this stage Virtual Design is not the only option, however it is a significant step forward within the field of QA.

Perhaps the final end result of Virtual Design Engineering is that all systems will be entirely designed within the virtual world, bringing the ability to test infinite variables and fully generate fault trees automatically. It will not necessarily be up to the quality assurance engineer's experience and creativity to come up with potential hazards, but rather a computer algorithm only to be verified by the human operator if deemed necessary. Like so many other professions, could the field of QA be overwhelmingly taken over by computers and software simulation? Only computing power and creativity hold back this vision of the future, and with each passing day these barriers come closer to coming down. Virtual Design Engineering and the VRQA model into the traditional quality assurance processes could help future system designers avoid the same engineering and managerial pitfalls uncovered back on that cold January day. 


\section{Acknowledgments}

We would like to express our sincere appreciation to the anonymous referees for their valuable comments and suggestions, which have enhanced the quality of the paper. 


\section{References}

Chou, C.-H., \& Üllrich, P. (2013). Fisker reduces number of prototypes, cuts timeto market with Virtual Performance Solution. Retrieved from http://articles.sae.org/11735/

Ericson, C. A. (2005). Hazard Analysis Techniques for System Safety. Hoboken,NJ, USA: John Wiley \& Sons, Inc. doi: 10.1002/0471739421

Hudspeth, M. (2005). MCAD MODELING METHODS--Virtual PrototypingDesign, test and present your product virtually to save time and money. CADalyst, 22(8), 42-44.

Jenab, K., \& Pineau, J. (2015). Failure Mode and Effect Analysis on Safety Critical Components of Space Travel. Management Science Letters, 5(7), 669-678. doi: 10.5267/j.msl.2015.5.006

Kenyon, A., van Rosendale, J., Fulcomer, S., \& Laidlaw, D. (2014, March 29). The design of a retinal resolution fully immersive VR display. VirtualReality (VR), 89-90. doi: 10.1109/VR.2014.6802065

Knight, J. F., \& Baber, C. (2007). Effect of Head-Mounted Displays on Posture.The Journal of the Human Factors and Ergonomics Society, 49(5), 797-807. doi: 10.1518/001872007X230172

Koopman, P. (2011). Using a Risk Analysis Table to Categorize Bug Priority. Retrieved from http://betterembsw.blogspot.com/2011/03/using-riskanalysis-table-to-categorize.html

Lerner, E. K., \& Lerner, B. W. (2013). Software and Hardware (2nd ed., Vol. 2). Detroit, MI, USA: Macmillan Reference USA.

McGarry, L., Qu, K. Y., Lee, W., \& Wilson, T. G. (2005). Quality assurance verification using a virtual prototyping system. Applied Power Electronics Conference and Exposition. 2, pp. 1325-1331. Austin: APEC. doi: 10.1109/APEC.2005.1453181

Mujber, T. S., Szecsi, T., \& Hashmi, M. J. (2004). Virtual reality applications in manufacturing process simulation. Journal of Materials Processing Technology, 155-156, 1834-1838. doi: 10.1016/j.jmatprotec.2004.04.401 
NASA. (1986). Report of the Presidential Commission on the Space Shuttle Challenger Accident. U.S. Government Printing Office. Retrieved from http://history.nasa.gov/ rogersrep/genindex.htm

Nesbitt, A. (2013). Virtual Prototyping. Appliance Design , 61 (5), 22-24. Retrived from http://www.appliancedesign.com/articles/93590-virtualprototyping

Oculus VR. (n.d.). Oculus Rift - Virtual Reality Headset for Immersive 3D Gaming. Retrieved from https://www.oculus.com/rift/

Rausand, M. (2005). Preliminary Hazard Analysis. Retrieved from LHC Beam Loss Monitoring System: https://ab-div-bdi-bl-blm.web.cern.ch/ab-divbdi-bl-blm/Literature/fmcea/pha.pdf

Redmond, P. (2007). A System of Systems Interface Hazard Analysis Technique. Monterey, CA, USA: Naval Postgraduate School. Retrived from file://C:/Users/kouroush/Downloads/ADA467343.pdf

Robertson, G. G., Card, S. K., \& Mackinlay, J. D. (1993). Three views of virtual reality: nonimmersive virtual reality. IEEE COMPUTER, 26 (2), 81-83. doi: 10.1109/2.192002

Rogers Commission. (1986). Report of the Presidential Commission on the Space Shuttle Challenger Accident. Washington, DC: U.S. Government Printing Office.

Safeware Engineering Corporation. (2003). Safeware Engineering Corp. Retrieved from http://www.safeware-eng.com/ Safety\%20White\%20Papers/System\%20Hazard\%20Analysis.htm

Takashi, S. (2002). Head mounted display. Displays , 23 (1-2), 57-64. doi:10.1016/S0141-9382(02)00010-0

TWI. (2014). FAQ: What is virtual prototyping? Retrieved from http://www.twiglobal.com/technical-knowledge/faqs/process-faqs/faq-what-is-virtualprototyping/

Vesely, B. (2005). Fault Tree Analysis (FTA): Concepts and Applications. Retrieved from: http://www.hq.nasa.gov/office/codeq/risk/docs/ftacourse.pdf 